\title{
Two-Stage Linearization Filter for Direct-Detection Subcarrier Modulation
}

\author{
Zhe Li, Student Member, IEEE, M. Sezer Erk1lınç, Member, IEEE, Robert Maher, Senior Member, IEEE, Lidia \\ Galdino, Member, IEEE, Kai Shi, Member, IEEE, Benn C. Thomsen, Member, IEEE, Polina Bayvel, Fellow, IEEE, \\ and Robert I. Killey, Member, IEEE
}

\begin{abstract}
A novel digital two-stage linearization filter is proposed for direct-detection (DD) systems and assessed experimentally for the first time. The performance improvement is quantified by experiments with a $7 \times 25 \mathrm{~Gb} / \mathrm{s}$ WDM DD single sideband 16-QAM Nyquist-shaped subcarrier modulation (Nyquist-SCM) system with a net optical information spectral density of $2.3(\mathrm{~b} / \mathrm{s}) / \mathrm{Hz}$. The results indicate that this technique can effectively compensate the nonlinearity caused by square-law detection while, at the same time reducing the DSP complexity, avoiding the need to perform the multiple iterations which are required in previously proposed beating interference cancellation methods.
\end{abstract}

Index Terms-Direct detection, Nyquist-pulse shaped subcarrier modulation, signal-signal beat interference cancellation, spectrally-efficient wavelength division multiplexing.

\section{INTRODUCTION}

QHORT- and medium-haul optical links/networks with increased throughputs are needed to meet the continuously increasing traffic demand. However, it is not currently practical to install coherent technologies in such applications due to their cost-sensitive nature [1]. Thus, it is still favorable to employ simpler direct-detection (DD) wavelength division multiplexing (WDM) systems for many inter-data center, access, metro and back-haul applications [2].

In DD applications, single sideband subcarrier modulation (SSB SCM) can be employed to achieve high information spectral densities (ISDs). Two SCM approaches are being studied for DD systems: optical orthogonal frequency division multiplexing (OFDM) [3] and Nyquist-pulse shaped subcarrier modulation (Nyquist-SCM) [4-7]. However, a nonlinear effect known as signal-signal beat interference (SSBI) results from the square-law detection and significantly degrades the system performance. Recently, a number of digital SSBI cancellation techniques have been demonstrated. Both the iterative symbol pre-distortion [8,9] and iterative post-compensation [10-11]

Manuscript received XXX. This work has been supported by the EU ERA-NET+ project, UK EPSRC UNLOC EP/J017582/1 project, EU FP7 ASTRON project and Semtech Corporation.

The authors are with the Optical Networks Group, Department of Electronic \& Electrical Engineering at University College London, WC1E 7JE, U.K. (e-mails: zhe.li@ee.ucl.ac.uk; m.erkilinc@ee.ucl.ac.uk; r.maher@ucl.ac.uk; lgaldino@ee.ucl.ac.uk; k.shi@ucl.ac.uk; b.thomsen@ucl.ac.uk; p.bayvel@ucl.ac.uk; r.killey@ucl.ac.uk). techniques require multiple iterations, thus significantly increasing the digital signal processing (DSP) complexity. The single-stage linearization filter proposed in [12] can reduce SSBI with a very simple DSP structure, but cannot achieve the maximum compensation gain due to the introduction of extra beating interference by the technique itself; its compensation performance can be improved by repeating the single-stage linearization filter iteratively (four times or more) and using the stored received signal waveform [7]. A single-stage linearization filter combined with single-iteration SSBI cancellation $[13,14]$ avoids the need for multiple iterations but still has the drawback of high dependency on the accuracy of symbol decision making.

In this letter, we propose a novel digital receiver-based two-stage linearization filter (a nonlinear filter) for DD SSB $\mathrm{SCM}$ systems. By implementing this technique, the SSBI is very effectively compensated without the need to perform multiple iterations of signal modulation and demodulation in the compensation DSP, which consequently leads to significantly lower complexity. We demonstrate the proposed scheme in experiments, showing that the proposed technique provides the highest compensation gain among the techniques being compared for both back-to-back and transmission assuming a hard-decision forward error correction (HD-FEC) bit error ratio threshold of $3.8 \times 10^{-3}$.

\section{Principle of TwO-Stage LinEARIZATION FILTER}

The proposed technique can be used for beating interference cancellation in both OFDM and Nyquist-SCM signaling. It consists of two stages. The first stage initially improves the performance by compensating the SSBI, and subsequently, the majority of the unwanted beating interference terms introduced by the first stage are eliminated in the second stage.

At the transmitter, the SSB SCM signal (OFDM or Nyquist-SCM) field, $E_{0}(n)$, is first generated, where $n$ is the discrete time index. In order to mitigate the accumulated dispersion during fibre transmission and low-pass filtering effects due to the limited bandwidth of the electrical components, chromatic dispersion pre-compensation (CDP) [15] and pre-emphasis are performed digitally. Following this, the real-valued optical carrier, $E_{\text {carrier }}$ is added during the electrical to optical conversion. After transmission and square-law detection, the normalized detected double-sideband $(\mathrm{DSB})$ signal, $V_{D D}(n)$ can be written as: 


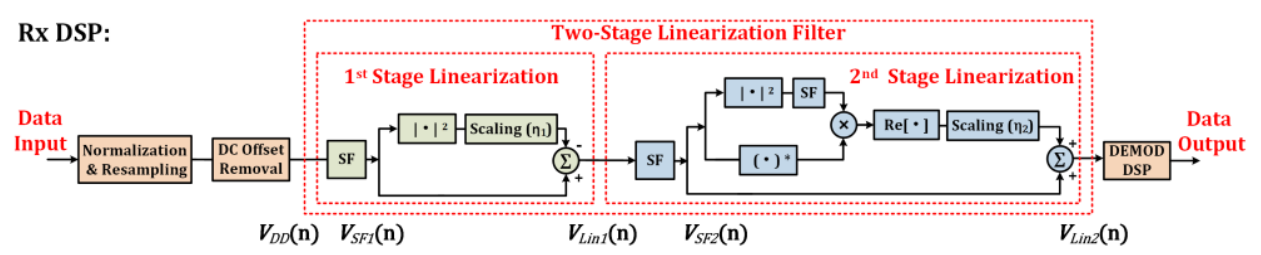

Fig.1: Receiver DSP with two-stage linearization filter. SF: sideband filter; DEMOD DSP: SCM signal demodulation.

$$
\begin{aligned}
V_{D D}(n) & =\left|E_{\text {carrier }}+E_{0}(n)\right|^{2} \\
& =E_{\text {carrier }}^{2}+2 \operatorname{Re}\left[E_{\text {carrier }} \cdot E_{0}(n)\right]+\left|E_{0}(n)\right|^{2}
\end{aligned}
$$

where $\operatorname{Re}[x]$ signifies the real part of $x$. In the right-hand side (RHS) of this equation, the first term is the direct current (DC) component, the second and third terms are the desired carrier-signal beating products (CSBP) and the SSBI products, respectively. Fig. 1 shows the receiver DSP (Rx DSP) structure with the two-stage linearization filter. In the first stage, following the DC removal and sideband filtering (SF), which is performed by applying an ideal digital Hilbert transform filter, a digital representation of the filtered SSB signal $\left(V_{S F I}(n)\right)$ after square-law detection is calculated, which is then subtracted from the original signal. The first stage output, $V_{\text {Linl }}(n)$ can be described as follows:

$$
\begin{gathered}
V_{S F 1}(n)=\alpha \cdot E_{0}(n)+\Lambda\left[\left|E_{0}(n)\right|^{2}\right] \\
V_{L i n 1}(n)=V_{S F 1}(n)-\eta_{1} \cdot\left|V_{S F 1}(n)\right|^{2} \\
=\alpha \cdot E_{0}(n)+\Lambda\left[\left|E_{0}(n)\right|^{2}\right]-\alpha^{2} \eta_{1} \cdot\left|E_{0}(n)\right|^{2} \\
-2 \alpha \eta_{1} \cdot \operatorname{Re}\left[E_{0}(n)^{*} \cdot \Lambda\left[\left|E_{0}(n)\right|^{2}\right]\right] \\
-\eta_{1} \cdot\left|\Lambda\left[\left|E_{0}(n)\right|^{2}\right]\right|^{2}
\end{gathered}
$$

where $\alpha$ is an amplitude scaling factor proportional to the value of the optical carrier-to-signal power ratio (CSPR), $\Lambda[\cdot]$ is the sideband filtering operator, and $\eta_{1}$ is the scaling parameter which controls the first stage linearization filter's effectiveness. In the RHS of equation (3), the first term is the desired SSB CSBP. Since only the positive frequency signal spectrum is demodulated, with the optimum adjustment of $\eta_{1}$, the second term (SSBI) is cancelled by the third term, the remaining terms are the signal-SSBI (fourth) and SSBI-SSBI (fifth) beating interference, which are significantly lower than the SSBI penalty. Hence, the total amount of nonlinear distortion is significantly reduced with respect to the case without performing the first linearization stage. Note that the use of the SF avoids unwanted beating products which would otherwise be generated by the negative frequency components.

Following this, the signal passes through the second linearization stage to mitigate the signal-SSBI beating interference introduced by the first stage, which is written as:

$$
\begin{aligned}
V_{S F 2}(n)=\alpha \cdot E_{0}(n) & -2 \alpha \eta_{1} \\
& \cdot \Lambda\left[\operatorname{Re}\left[E_{0}(n)^{*} \cdot \Lambda\left[\left|E_{0}(n)\right|^{2}\right]\right]\right]-\eta_{1} \\
& \cdot \Lambda\left[\left|\Lambda\left[\left|E_{0}(n)\right|^{2}\right]\right|^{2}\right] \\
V_{L i n 2}(n)=V_{S F 2}(n) & +\eta_{2} \cdot \operatorname{Re}\left[V_{S F 2}(n)^{*} \cdot \Lambda\left[\left|V_{S F 1}(n)\right|^{2}\right]\right]
\end{aligned}
$$

where $V_{S F 2}(n)$ is the filtered SSB signal, $V_{\text {Lin } 2}(n)$ is the output of the second linearization stage. The scaling factor $\eta_{2}$ needs to be optimized to achieve the maximum compensation gain. The optimum values of the scaling factors $\left(\eta_{1}\right.$ and $\left.\eta_{2}\right)$ can be found by sweeping $\eta_{1}$ first and $\eta_{2}$ afterwards while measuring the corresponding BERs. As can be seen from Eqs. (3) and (5), the optimum scaling factor values have a high dependency on the applied CSPR value, and thus, need to be optimized for each utilized CSPR value. They both reduce when the CSPR value is increased. Since the input of the second stage, $V_{\text {LinI }}(n)$ is mainly the desired CSBP (SSBI is compensated in the first stage), the estimation of the signal-SSBI beating is significantly improved so that the majority of the signal-SSBI beating interference can be eliminated after this stage, which further improves the compensation performance. The SSBI-SSBI beating term results in a very small penalty with respect to the signal-SSBI beating term. In order to keep the DSP simple, this is left uncompensated. The proposed two-stage linearization filter requires more than twice the DSP hardware compared with the single-stage linearization filter [12] due to the use of extra sideband filters and multipliers. However, since all the compensation is carried out before the SCM signal demodulation (DEMOD DSP), there is no need for additional SCM signal demodulation and modulation stages, and hence, its complexity is significantly lower than the previously proposed techniques in $[10,13,14]$.

\section{EXPERIMENTAL SETUP}

The proposed scheme was assessed experimentally in a 7 channel WDM DD SSB 16-QAM Nyquist-SCM system using the optical test-bed shown in Fig. 2. First, the odd and even channels were generated using two IQ-modulators, which were driven by two digital-to-analog converters (DACs), operating at a sampling rate of $25 \mathrm{GSa} / \mathrm{s}$. In the transmitter DSP, a 25 $\mathrm{Gb} / \mathrm{s}$ (at a symbol rate $\left(f_{s}\right)$ of $6.25 \mathrm{GBd}$ ) SSB 16QAM Nyquist-SCM signal was generated using decorrelated $2^{15}-1$ de Bruijn sequences. Pulse shaping was carried out using root-raised cosine filters with a roll-off parameter of 0.1 , and the subcarrier frequency was chosen to be $0.55 \times f_{s}$. CDP was utilized following the modulation DSP in the case of fiber transmission. The WDM channel spacing was set to $10 \mathrm{GHz}$ to

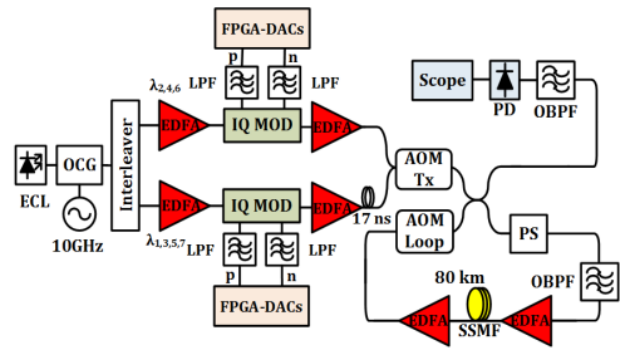

Fig. 2: Optical transmission experimental test-bed. ECL: External cavity laser, OCG: Optical comb generator, EDFA: Erbium-doped fiber amplifier, DAC: Digital-to-analog converter, LPF: Low-pass filter, AOM: Acousto-optic modulator, PS: Polarization scrambler, OBPF: Optical band-pass filter, PD: Photodiode. 
maintain the linear crosstalk penalty caused by neighboring channels at less than $1 \mathrm{~dB}$ and maximize the ISD employing the available components, offering a gross ISD of $2.5 \mathrm{~b} / \mathrm{s} / \mathrm{Hz}$, and the CSPR value was optimized to achieve the optimum system performance. The optical carrier was generated by biasing the IQ-modulators above the null point and the biases were adjusted to achieve the desired CSPR values. The method used to accurately measure the CSPR is described in [6].

A recirculating fiber loop, with a single span of $80 \mathrm{~km} \mathrm{SSMF}$ was used for transmission. Two erbium-doped fiber amplifiers (EDFAs) with a $5 \mathrm{~dB}$ noise figure were used to compensate the loop loss. Following transmission, the channel of interest was de-multiplexed at the receiver using a $10 \mathrm{GHz}$ bandwidth optical band-pass filter (OBPF), detected by a single-ended PIN photodiode, and subsequently, digitized with a single analog-to-digital converter (ADC) at $50 \mathrm{GSa} / \mathrm{s}$. Note that, although high oversampling DAC/ADC rates were utilized in the experimental demonstration, for practical system implementation, lower sampling rates with steeper anti-imaging and anti-aliasing filters could be implemented in the transmitter and receiver, respectively with little impact on the performance of the compensation scheme. Following detection and digitization, the proposed two-stage linearization filter was implemented in the receiver DSP, as shown in Fig. 1. Its compensation performance was compared with the receiver-based iterative SSBI post-compensation [10] using hard decision decoding with rectangular decision boundaries and single-stage linearization filter [12]. To achieve the maximum compensation gain using the iterative scheme, four iterations were performed.

\section{RESUlTS AND DISCUSSION}

\section{A. Optical Back-to-back Performance}

The optical back-to-back performance evaluation was carried out by performing amplified spontaneous emission (ASE)-noise loading at the receiver to measure the BER as a function of OSNR (measured with $0.1 \mathrm{~nm}$ resolution bandwidth, and the $25 \mathrm{~Gb} / \mathrm{s}$ signal power in the measurement includes both the sideband signal and the optical carrier), as shown in Fig. 3(a) for the cases without and with SSBI mitigation. The CSPR value was swept from $6 \mathrm{~dB}$ to $14 \mathrm{~dB}$ and adjusted at each OSNR level to achieve the optimum system

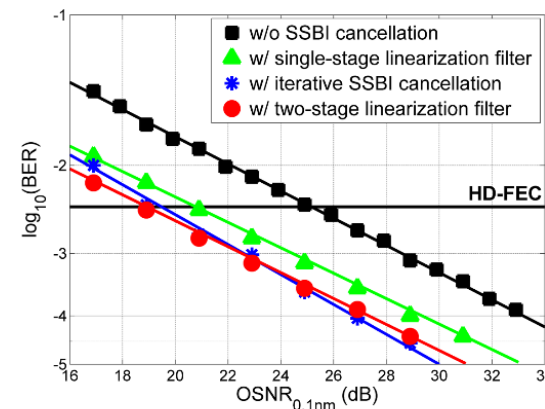

(a)

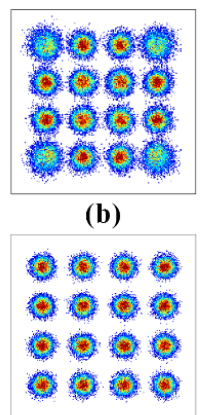

(c)
Fig. 3: (a) BER versus OSNR for cases without and with the iterative SSBI cancellation, single-stage and two-stage linearization filters (left). The received constellation diagrams at an OSNR of $29 \mathrm{~dB}$ (right), (b) without $(\mathrm{EVM}=16.6 \%)$ and with $(\mathrm{EVM}=12.6 \%)$ two-stage linearization filter.

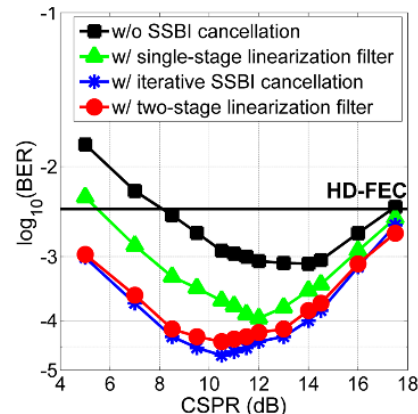

(a)

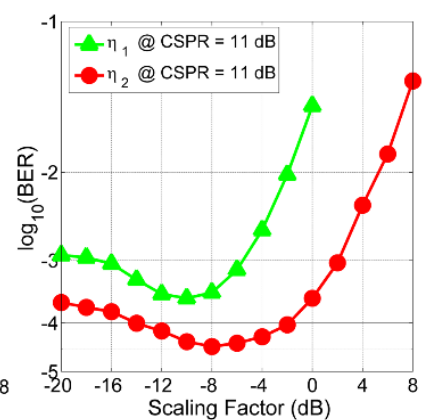

(b)
Fig. 4: (a) BER versus CSPR without and with iterative SSBI cancellation, single- and two-stage linearization filters at OSNR $=29 \mathrm{~dB}$. (b) BER versus scaling factors $\left(\eta_{1}\right.$ with $\eta_{2}=0$, and $\eta_{2}$ with $\eta_{1}$ set to the optimum value) with two-stage linearization filter at OSNR $=29 \mathrm{~dB}, \mathrm{CSPR}=11 \mathrm{~dB}$.

performance. For every $2 \mathrm{~dB}$ increase in OSNR, the optimum CSPR value increases by approximately $1 \mathrm{~dB}$. The required OSNR at the HD-FEC threshold (assumed to be BER $=3.8 \times$ $10^{-3}$ ) was found to be $25.3 \mathrm{~dB}$ without SSBI compensation, with an improvement by $6.4 \mathrm{~dB}$ to $18.9 \mathrm{~dB}$ for the case with the proposed two-stage linearization filter, which is higher than the $6.0 \mathrm{~dB}$ and $4.3 \mathrm{~dB}$ gains achieved using the iterative SSBI cancellation and single-stage linearization filter, respectively. As described in Section II, the proposed two-stage linearization filter further eliminates the majority of the introduced beating interference introduced by the single-stage linearization filter, and provides significantly improved performance. At the same time, since its performance does not depend on the accuracy of symbol decisions, it offers slightly better performance than the iterative scheme at lower OSNRs. From Figs. 3(b) and 3(c), it can be observed that after implementing the two-stage linearization filter, the compensated constellation is significantly less distorted than the uncompensated one, especially symbols on the periphery, which are the most affected by the SSBI due to their higher symbol energies.

Due to the reduction in the SSBI, the optimum CSPR value was reduced by $2 \mathrm{~dB}$, compared to the case without SSBI compensation, to achieve the maximum compensation gain when using the single-stage linearization filter and by approximately $3 \mathrm{~dB}$ with the two-stage linearization filter. The optimization of CSPR values without and with SSBI cancellation is shown by the BER versus CSPR plots without and with SSBI mitigation schemes at 29 dB OSNR in Fig. 4(a). The BER versus scaling factors $\left(\eta_{1}\right.$ and $\left.\eta_{2}\right)$ applied in the two-stage linearization filter at $29 \mathrm{~dB}$ OSNR and $11 \mathrm{~dB}$ CSPR is shown in Fig. $4(\mathrm{~b})\left(\eta_{\mathrm{dB}}=10 \times \log _{10}\left(\eta_{\text {linear }}\right)\right.$. The BER is initially reduced by the optimum adjustment of scaling factor $\eta_{1}$ (with $\eta_{2}$ set to zero), following which it is further decreased by optimizing $\eta_{2}$. The plot shows the sensitivity of the system to the scaling factor values. The optimum value of $\eta_{1}$ is found to be approximately $2 \mathrm{~dB}$ lower than $\eta_{2}$.

\section{B. WDM Transmission Performance}

Following the back-to-back performance evaluation, WDM transmission experiments over distances of up to $800 \mathrm{~km}$ of uncompensated SSMF were performed for cases without and with the SSBI cancellation approaches. The following results 


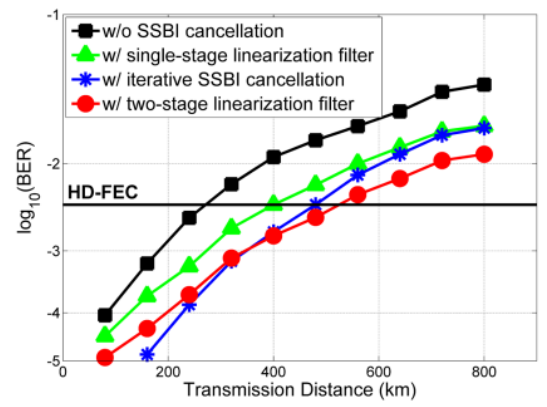

Fig. 5: BER versus transmission distance for cases without and with iterative SSBI cancellation, single- and two-stage linearization filters.

exhibit the performance of the center WDM channel. The measured BER values with respect to the transmission distances are plotted in Fig. 5 at the optimum CSPR and optical launch power. The OSNR values range from $36 \mathrm{~dB}$ for $80 \mathrm{~km}$ transmission to $24 \mathrm{~dB}$ at $800 \mathrm{~km}$, and the optimum CSPR correspondingly decreases from $16 \mathrm{~dB}$ to $11 \mathrm{~dB}$. It shows that the transmission performance is significantly improved at all distances by implementing the SSBI mitigation schemes. For WDM transmission distances of up to $400 \mathrm{~km}$, the iterative scheme offers the best compensation performance due to the accurate SSBI estimation. However, its performance degrades at longer distances at which the OSNR is lower, as the back-to-back results shown in Fig. 3 suggest. For distances of more than $400 \mathrm{~km}$, the compensation performance of the two-stage linearization filter surpasses the iterative scheme and provides the best performance. The obtained BER values of the two-stage linearization filter are almost halved in contrast to the single-stage linearization filter.

In order to further assess the transmission performance at different optical launch powers, the WDM system transmission performance at $480 \mathrm{~km}$ was evaluated by plotting the BER versus optical launch power, as shown in Fig. 6. The CSPR was optimized to $13 \mathrm{~dB}$ without and $10-11 \mathrm{~dB}$ (depending on the SSBI compensation technique being used) with SSBI cancellation techniques at the optimum launch power per channel and kept the same for all the optical launch power values. It can be observed that the BER at the optimum launch power was reduced from $1.6 \times 10^{-2}$ to $2.7 \times 10^{-3}$ using the two-stage linearization filter, which offers the best performance among these techniques. Furthermore, the BER values for all the seven channels after implementing two-stage linearization

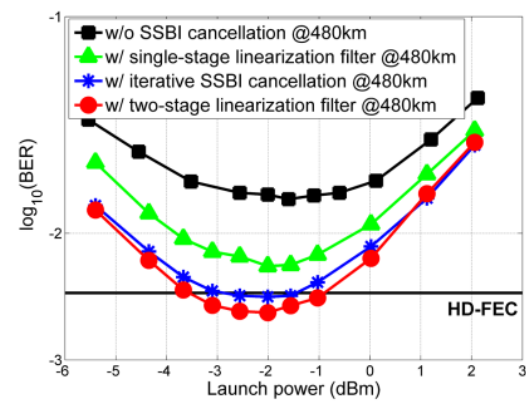

Fig. 6: (a) BER versus optical launch power per $25 \mathrm{~Gb} / \mathrm{s}$ channel at $480 \mathrm{~km}$ WDM transmission for cases without and with iterative SSBI cancellation, single- and two-stage linearization filters. filter was measured to be between $2.4 \times 10^{-3}$ and $3.2 \times 10^{-3}$, which, based on the standard 7\% HD-FEC threshold of $3.8 \times$ $10^{-3}$, results in a net optical ISD of $2.3(\mathrm{~b} / \mathrm{s}) / \mathrm{Hz}$.

\section{CONCLUSIONS}

In this letter, we proposed and experimentally assessed, for the first time, a novel digital two-stage linearization filter for direct-detection single-sideband subcarrier modulation systems. Its compensation performance was experimentally assessed on a $10 \mathrm{GHz}$-spaced $7 \times 25 \mathrm{~Gb} / \mathrm{s}$ WDM single-sideband 16-QAM Nyquist-SCM direct-detection system. The results suggest that the proposed technique provides the highest compensation gain among the techniques being compared at the $3.8 \times 10^{-3}$ HD-FEC threshold. Furthermore, since the proposed technique does not require multiple iterations of interference cancellation, the DSP complexity is relatively simple.

\section{REFERENCES}

[1] D. Che, Q. Hu, and W. Shieh, "Linearization of direct detection optical channels using self-coherent subsystems," J. Lightw. Technol., vol. 34, no. 2, pp. 516-524, 2016.

[2] R.I. Killey, M.S. Erkılınç, Z. Li, S. Pachnicke, H. Griesser, R. Bouziane, B.C. Thomsen, and P. Bayvel, "Spectrally-efficient direct-detection WDM transmission system," in Proc. ICTON, 2015, paper We.B3.2.

[3] B.J.C. Schmidt, A.J. Lowery, and L.B. Du, "Low sample rate transmitter for direct-detection optical OFDM," in Proc. OFC, 2009, paper OWM4.

[4] A.O. Wiberg, B.-E. Olsson, and P.A. Andrekson, "Single cycle subcarrier modulation," in Proc. OFC, 2009, paper OTuE.1.

[5] J.C. Cartledge and A.S. Karar, " $100 \mathrm{~Gb} / \mathrm{s}$ intensity modulation and direct detection," J. Lightw. Technol., vol. 32, no. 16, pp. 2809-2814, 2014.

[6] M.S. Erkılınç, Z. Li, S. Pachnicke, H. Griesser, B.C. Thomsen, P. Bayvel, and R.I. Killey, "Spectrally-efficient WDM Nyquist-pulse-shaped 16-QAM subcarrier modulation transmission with direct detection," $J$. Lightw. Technol., vol. 33, no. 15, pp. 3147-3155, 2015.

[7] K. Zou, Y. Zhu, F. Zhang and Z. Chen, "Spectrally efficient terabit optical transmission with Nyquist 64-QAM half-cycle subcarrier modulation and direct-detection," Opt. Lett., vol. 41, no. 12, pp. 2767-2770, 2016.

[8] C. Sánchez, B. Ortega, and J. Capmany, "System performance enhancement with pre-distorted OOFDM signal waveforms in IM/DD systems," Opt. Express, vol. 22, no. 6, pp. 7269-7283, 2014.

[9] C. Ju, X. Chen, N. Liu, and L. Wang, "SSII cancellation in 40 Gbps VSB-IMDD OFDM system based on symbol pre-distortion," in Proc. ECOC, 2014, paper P.7.9.

[10] W.R. Peng, X. Wu, K. Feng, V.R. Arbab, B. Shamee, J. Yang, L. C. Christen, A. E. Willner, and S. Chi, "Spectrally efficient direct-detected OFDM transmission employing an iterative estimation and cancellation technique," Opt. Express, vol. 17, no. 11, pp. 9099-9111, 2009.

[11] Z. Li, M. S. Erkılınç, S. Pachnicke, H. Griesser, R. Bouziane, B.C. Thomsen, P. Bayvel, and R.I. Killey, "Signal-signal beat interference cancellation in spectrally-efficient WDM direct-detection Nyquist-pulse-shaped 16-QAM subcarrier modulation," Opt. Express, vol. 23 , no. 18 , pp. 23694-23709, 2015.

[12] S. Randel, D. Pilori, S. Chandrasekhar, G. Raybon, and P. Winzer, "100-Gb/s discrete-multitone transmission over $80-\mathrm{km}$ SSMF using single-sideband modulation with novel interference-cancellation scheme," in Proc. ECOC, 2015, paper Mo.4.5.2.

[13] Z. Li, M. S. Erkilinc, R. Bouziane, R. Maher, L. Galdino, K. Shi, B. C. Thomsen, P. Bayvel, and R. I. Killey, "Simplified DSP-based signal-signal beat interference mitigation for direct detection subcarrier modulation", in Proc. OFC, 2016, paper W1A.3.

[14] Z. Li, M. S. Erkilinc, R. Maher, L. Galdino, K. Shi, B. C. Thomsen, P. Bayvel, and R. I. Killey, "Reach enhancement for WDM direct-detection subcarrier modulation using low-complexity two-stage signal-signal beat interference cancellation", in Proc. ECOC, 2016, paper M.2.B.1.

[15] R.I. Killey, P.M. Watts, V. Mikhailov, M. Glick, and P. Bayvel, "Electronic dispersion compensation by signal predistortion using digital processing and a dual-drive Mach-Zehnder modulator," IEEE Photon. Technol. Lett., vol.17, no. 3, pp. 714-716, 2005. 